\title{
Novel method for watermarking system operating on the HF and VHF radio links
}

\author{
Z. Piotrowski \& P. Gajewski \\ Electronics Faculty, Military University of Technology, Poland
}

\begin{abstract}
Modern watermarking systems operating on an acoustic layer of the host signal must meet a lot of constructional requirements. One of these requirements is watermark perceptual transparency at host signal presence and robustness to intentional and unintentional attacks. Developed systems also possess a dedicated bit rate for the steganographic mode or a data payload for the watermarking mode. The system presented in this paper relates to the watermarking systems class with blind detection procedure and operates on the host speech signal transmitted over HF/VHF radio links. System innovation consists of using the OFDM and SSDS techniques as well as a drift phase scanner on the receiver's side. The system was developed for radio correspondent authorisation using HF/VHF radio links.

Keywords: watermarking, steganography, information hiding, phase drift scanner, radio HF/VHF link, OFDM signal, Spread Spectrum Direct Sequence, coherent averaging, synchronization.
\end{abstract}

\section{Introduction}

The radio correspondent authorization problem in the HF/VHF links is very important for the reason that the degraded and distorted speaker's recognition signal is basic authorisation element for the radio end-user. Authorisation is carried out "by ear" in a subjective manner, e.g. radio operator, using radio handset, assesses that his/her interlocutor possesses characteristic speech features and qualifies recognised speech to dedicated person. It is typical that after "short, subjective authorisation" the radio speech correspondence takes place usually using secret messages not accessible for third party users or outsiders. The subjective authorisation problem is more complicated when we take into 
consideration the abilities of the modern vocoders technique: real time speech analysis and synthesis using vocal tract and pitch signal dedicated for one speaker and transformed to another one using the same speech features: tone quality, timbre, intonation, pitch, vocal tract parameters distribution etc. Synthesized speech quality and fidelity as well as intelligibility is 'almost the same' for the radio link end-user has good hearing (Human Auditory System with normal sensitivity). The radio correspondent authorization process so far, is based on open, public mechanisms of the ad hoc query and transient response using standard LCD radio display to indicate authorisation process. The authorisation data stream allocated into standard radio protocol frames (blocks) allows for effective transmission of the query-response block sentences using a dedicated radio and terminal developed and proposed by a producer.

The mechanism of the hidden authorization of the radio correspondent presented in this paper is free from following disadvantages:

- open, public query and response (authorization status displayed on LCD, signalling using buzzer etc.)

- authorisation protocol defined by a producer

- authorisation process not standardized for all telecommunication equipment, dedicated equipment only (radio model etc.)

In the proposed system, the authorisation process takes place during the radio call at the host speech signal background. The system does not interfere either in the hardware or software radio station. The speech signal processed in the software watermark coder is passed to the radio station's input (standard handset socket). The input speech signal is processed - this means that the binary correspondent's Personal Identification Number represented by the binary signature is mapped in the frequency domain and a psychoacoustic corrected watermark is embedded into the host speech signal). The watermark in the host speech signal presence is perceptually transparent for the Human Auditory System (HAS).

\section{Watermarking system}

The developed system consists of a software watermark coder (embedder) and a decoder (extractor). Implementation was carried out in the Matlab scientific programming environment. The system process speech signal has a sampling frequency $\mathrm{fs}=48 \mathrm{kHz}$ and resolution 16 bits/sample. A two-way impedance commutator is used on the transmitters as well as the receiver's side to switch over radio simplex mode: transmitting or receiving. Watermark embedding and extracting uses the off-line mode (not real-time processing).

\subsection{Watermark embedding}

A basic block scheme of the watermark coder is shown in fig. 1 .

The watermark coder consists of two modulators: OFDM as well as BPSK SSDS. The OFDM modulator generates a watermark pattern based on mapped information in accordance with the PIN binary sequence. The OFDM mapper 
allocates successive bits into individual frequency bins, in this way the OFDM pass band occupies the $6^{\text {th }}$ and $37^{\text {th }}$ frequency bins (FFT length 512 points and $\mathrm{fs}=48 \mathrm{kHz}$ ):

$$
\frac{f_{s}}{F F T_{\text {length }}} \times 6=562,5 \mathrm{~Hz} \text {, and } \frac{f_{s}}{F F T_{\text {length }}} \times 37=3468,75 \mathrm{~Hz}
$$

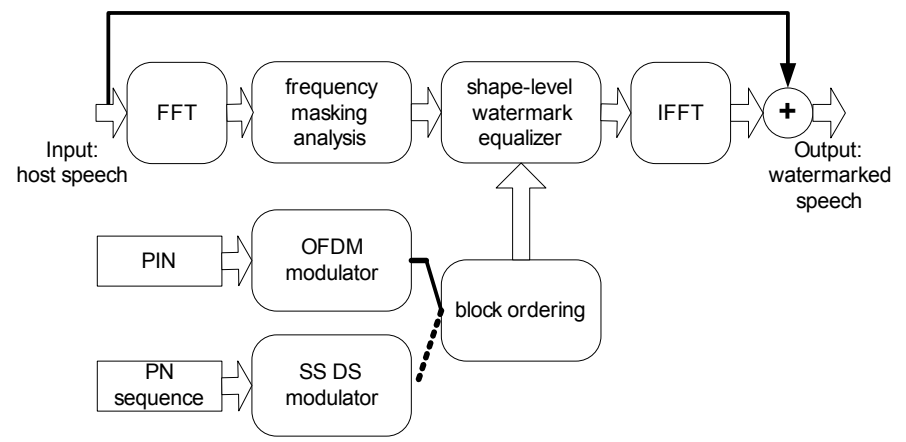

Figure 1: Basic block scheme of the watermark coder.

Watermark data payload amounts are for the base block sequence (one frame, block) 16 bits. Each successive bit is represented by orthogonally distributed spectral pair lines $\alpha_{i}, \alpha_{i+1}$. Logical bit "1" is mapped based on the following spectral line amplitude value rule: $\alpha_{i}>\alpha_{i+1}$, and for logical bit "0": $\alpha_{i}<\alpha_{i+1}$. In the described system only two binary values are assumed: $\alpha \in\{0,1\}$. The information mapper work principle for the OFDM modulator is shown in fig. 2.

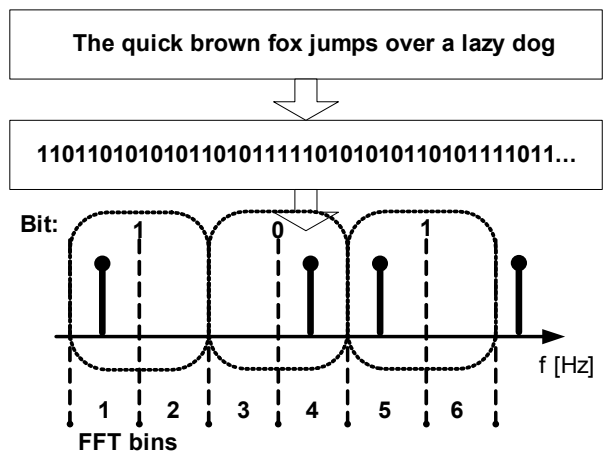

Figure 2: $\quad$ OFDM information mapper - work principle.

The six chosen spectral lines are considered as spectral phase "pilots" used on the receiver's side for the output phase drift estimation $\Phi_{\Delta}$ of the radio link. The 
SSDS signal based on the preliminary BPSK modulator is used for synchronisation of the watermark sequence. The following parameters are assumed: conversion gain $S N R_{S S D S}=24,77 d B$, spread spectrum pass band: $B_{s}=3 \mathrm{kHz}$. A spreading signal is generated using a pseudorandom information sequence. Depending on the assumed block data format, a system can be considered as steganographic (OFDM sequence for data block precedes by SSDS sequence block) or watermarking (each OFDM block with the same data payload in the stream). A steganographic system has a dedicated bit rate, a watermarking system is characterised by a data payload parameter ( 16 bits in the case of the described system).

The host signal is transformed to the frequency domain and psychoacoustic processing is performed using the Mpeg1 Layer1 algorithm to compute the Just Noticeable Difference threshold level (JND) as a difference between host level and the minimal masking threshold. The parameter Signal-to-Mask Ratio (SMR) is computed, separately for each critical sub-band (logarithmic Bark scale) for the watermark spectrum range. The SMR parameter is used for the watermark correction procedure (block: shape-level watermark equalizer) to ensure watermark signal perceptual transparency at the host speech signal presence. The corrected watermark in frequency shape and level is transformed back to the time domain using IFFT and summed together with the host signal. The developed system is based on direct host signal summing with corrected watermark, unlike the system described in [1] where the mean value of the host signal's FFT magnitudes in the dedicated sub-band is computed to add the watermark spectrum line. An example of the watermarked signal spectrum is shown in fig. 3 .

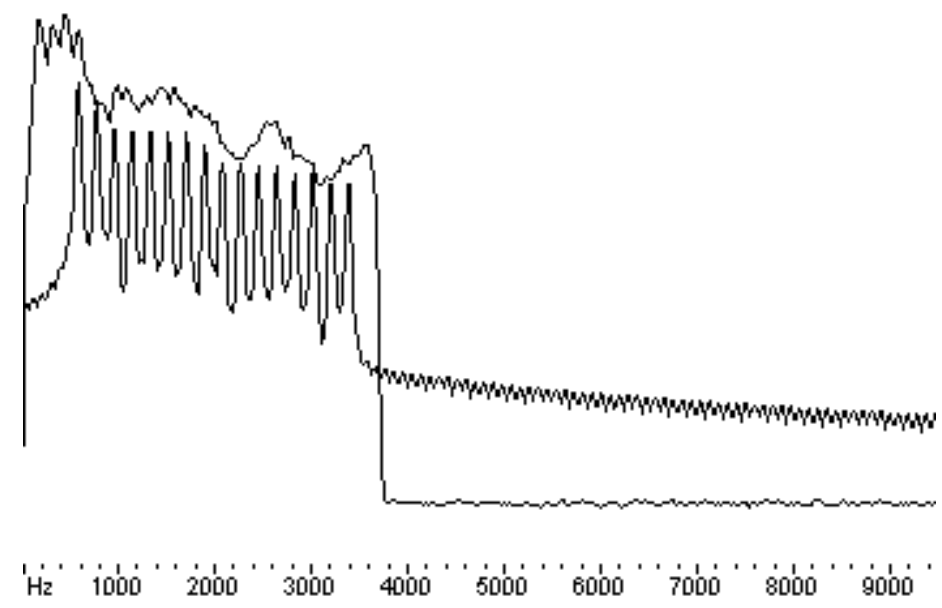

Figure 3: Example of the watermarked signal spectrum (host speech signal) and hidden (JND level) watermark. 


\subsection{Watermark decoding}

The basic block scheme of the watermark decoder is shown in fig. 4 .

The watermark decoder consists of a synchronisation block. This block uses a SSDS demodulator. The demodulator generates the same PN binary sequence as on the transmitter side, a $2 \mathrm{kHz}$ carrier wave allows one to make a transformation from $500-3500 \mathrm{~Hz}$ into the base pass band. The decoding process based on correlation analysis finds synchronisation points for correct data block bit decoding. Optional LPC analysis (carried out in synchronizer block) is useful for the effective decoding process (the decorrelation procedure generates a residual signal with small standard deviation). Buffer delay delays the start of the data blocks for the decoding process. An example of a BPSK DS signal spectrum taking part in the decoding process is shown in fig. 5 .

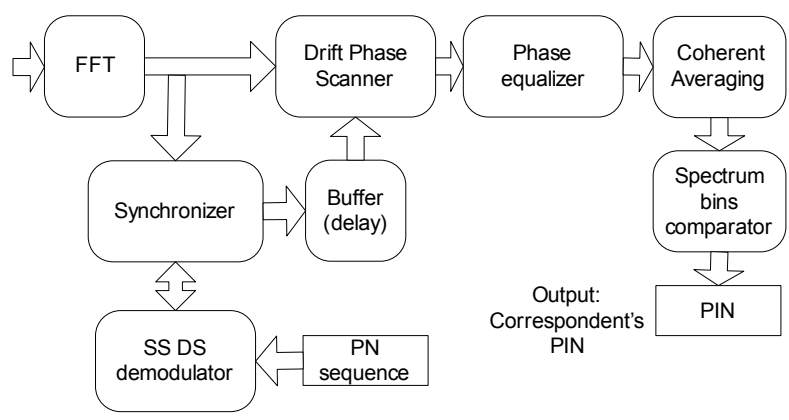

Figure 4: Basic block scheme of the watermark decoder.

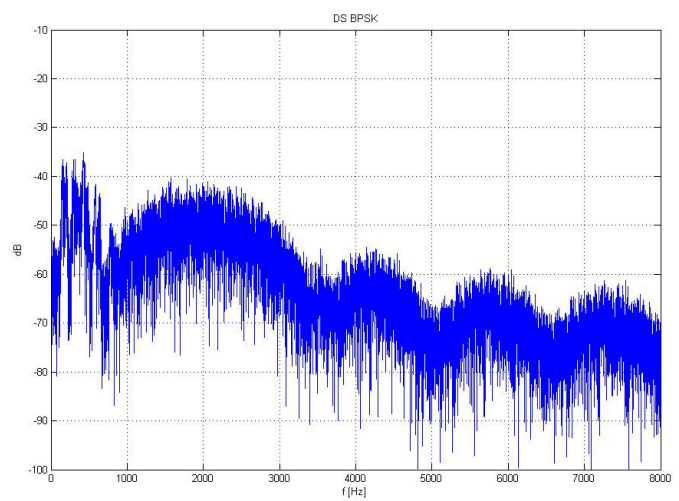

Figure 5: BPSK SSDS signal spectrum.

The synchronisation process is optional and useful only in the steganographic system case. The main goal of the projected algorithm is a watermarking system based on a drift phase scanner, a phase angle correction and a coherent averaging process. 
The drift phase scanner method was developed and described in detail in the $\mathrm{PhD}$ dissertation [2] and then published in [3]. The decoded signal has ultimate frequency detuning (phase drift) caused by phase drift $\mathrm{A} / \mathrm{C}$ and $\mathrm{C} / \mathrm{A}$ converters' (clock short-term stability) as well as output small stability heterodyne devices' (in transceiver radios). Spectrum coherention as a key requirement for proper spectrum averaging must be met to decrease host speech signal standard deviation and receive proper coherent gain:

$$
S N R_{c o h}=10 \cdot \log _{10}(M)(\mathrm{dB})
$$

where:

$M$ - iterations number (signal block averaging)

Example: signal duration equals 10 second, $\mathrm{fs}=48 \mathrm{kHz}$ and data block 512 samples gives: $S N R_{c o h}=19,72(\mathrm{~dB})$. This result means that the watermark signal "hidden" under the host speech signal at value $S M R<S N R_{c o h}$ is still possible to be correctly detected, of course, if coherence is met.

Spectrum averaging is based on the formula (2) for the chosen $n$ spectral line:

$$
F F T_{c o h}(n)=\frac{\sum_{i=1}^{N} \operatorname{Re}\left\{F F T_{i}(n)\right\}}{N}+j \frac{\sum_{i=1}^{N} \operatorname{Im}\left\{F F T_{i}(n)\right\}}{N}
$$

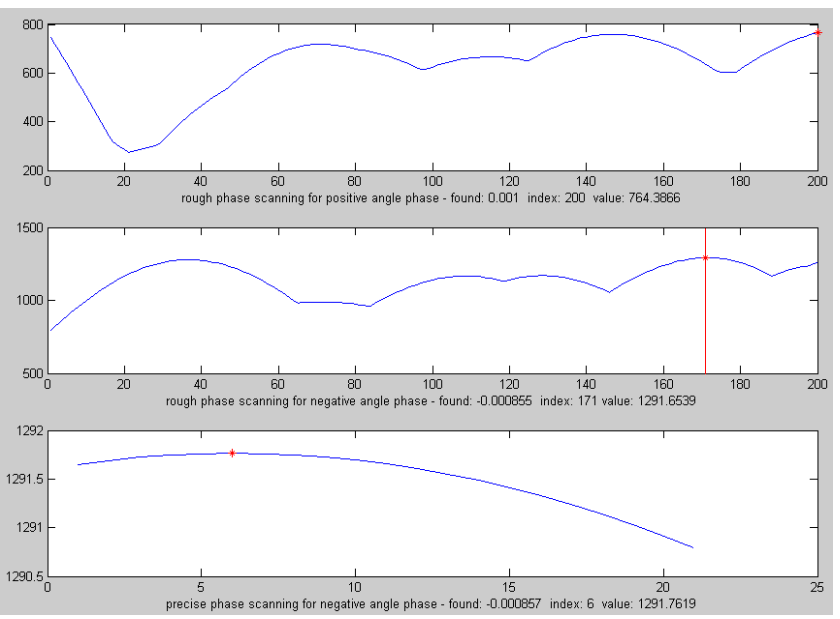

Figure 6: Example of searching radio link drift phase, result: $\phi=-8.57 \mathrm{e}-4$ $\mathrm{rad} / \mathrm{block}$.

The final result as a module for a complex number has a maximum value only in the case when the coherence requirement is met:

$$
\gamma_{\max }=\operatorname{abs}\left(F F T_{c o h}(n)\right)
$$


which means even a small drift value of the phase angle for dedicated spectral line during spectrum averaging will decrease the module value $\gamma$. The drift phase scanner searches the all angle range $\langle-\phi, \phi\rangle$ (limited for predicted output drift values and desired method resolution) to find the maximum value $\gamma_{\max }$. The searching process is based on virtual module computation. The virtual module is considered as a sum of modules for "pilots" spectral lines and is divided onto two stages: rough and precise searching (fig. 6, fig. 7). The output drift phase value found in the drift scanner is a base parameter in the block: phase equalizer. After the phase equalization process (phase value correction) the signal spectrum is averaged and spectrum bins are pairs' compared to find the output PIN binary sequence.

The watermarked host speech spectrum after coherent averaging after drift equalisation is shown in fig. 8 .

Input PIN signature on the transmitter side: [1111111111111111].

OFDM information mapper allocated energy to the frequency bins numbers: 7-38 (with DC component) in the following sequence: [10101010101010101010101010101010]. The PIN signature was decoded on the receiver's side with 0 errors using spectrum lines comparator.

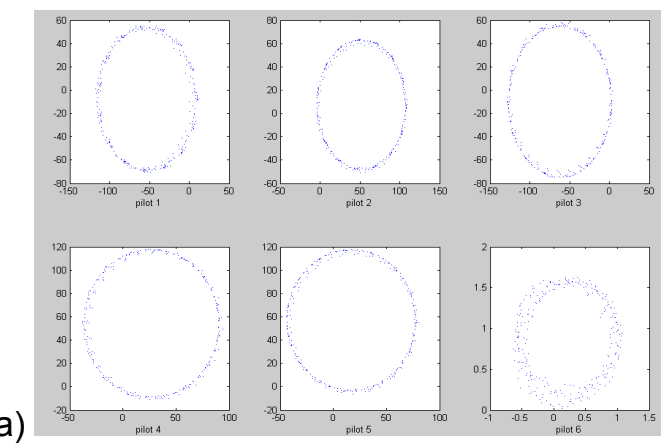

a)

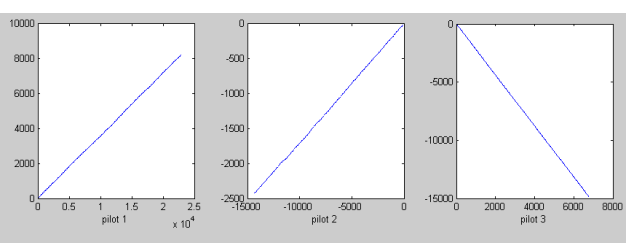

b)
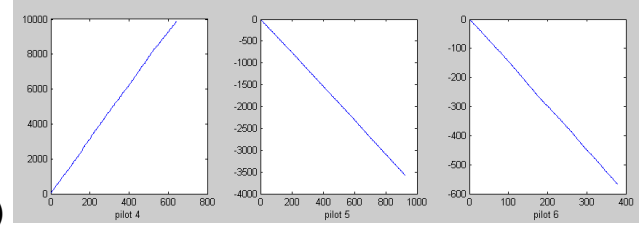

Figure 7: "Pilots" spectral lines before a) and after b) drift correction procedure Im-Re plots. 


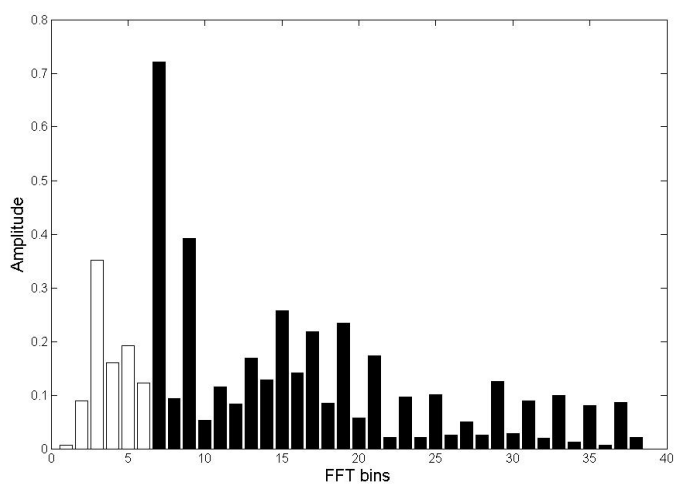

Figure 8: Watermarked host spectrum after coherent averaging.

\section{Experimental results}

Experiment number 1 was dedicated for perceptual transparency assessment and based on standard ITU-R BS 1116-1 subjective fidelity test. The full procedure and the environmental conditions that were required to be met were described in detail in [4]. Results for subjective assessment by listeners are shown in fig. 9.

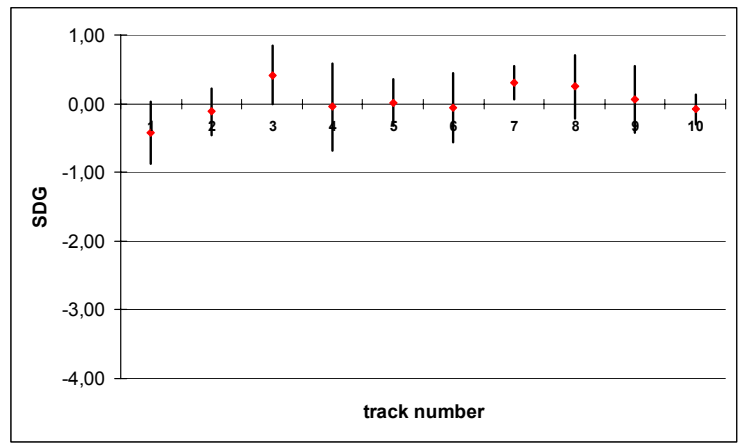

Figure 9: $\quad$ ITU-R BS 1116-1 results for 10 tracks.

Subjective fidelity assessment was done for 10 (male/female) speech tracks with an embedded watermark and degraded by HF/VHF noise. These tracks were compared with host speech tracks degraded also by HF/VHF noise. Every measurement point was characterized by 15 listeners with normal hearing (based on preliminary audiogram tests). Measurement results were taking into a statistical estimation procedure with a $95 \%$ confidence level. A SGD score above -1 SDG value shows that the embedded watermark is completely transparent at the host speech signal presence. A SDG positive score shows listeners better assessed the fidelity watermarked speech signal in comparison with the host speech signal. 
Experiment number 2 was dedicated for estimating of the decoding data payload efficiency. The computer simulated model of the HF/VHF radio channel degraded watermarked signal as well as the simulation of the radio simplex mode was taken into account. The result of the radio simplex mode simulation for the output received signal is a set of short signal fragments with various durations. These signal fragments are decoded separately (averaging complex spectrum and modules computation) and finally summed, as shown in table 1.

Experiment number 3 was dedicated for estimation of the decoding data payload efficiency in the real radio channel using a standard FM/AM modulator and a standard radio FM/AM receiver. The modulated watermarked signal (duration: 10 second) was transmitted over the radio channel and demodulated on the receiver's side and decoded correctly in PC software (0 errors / 16 bits correct decoded).

Table 1: $\quad$ PIN decoding efficiency with simulation of the radio simplex mode and HF/VHF channel.

\begin{tabular}{|l|c|c|c|c|}
\hline \multicolumn{1}{|c|}{ Track } & $\begin{array}{c}\text { Duration } \\
(\mathrm{s})\end{array}$ & $\begin{array}{c}\text { Track } \\
\text { Avg RMS } \\
\text { Power }(\mathrm{dB})\end{array}$ & $\begin{array}{c}\mathrm{SNR}^{*} \\
(\mathrm{~dB})\end{array}$ & $\begin{array}{c}\text { Decoder } \\
\text { error } / \\
\text { total }\end{array}$ \\
\hline Track - part 01 & 0.877 & -20.4 & 1.92 & $4 / 16$ \\
\hline Track - part 02 & 0.93 & -22.87 & 5.46 & $3 / 16$ \\
\hline Track - part 03 & 0.922 & -22.65 & 6.54 & $5 / 16$ \\
\hline Track - part 04 & 1.181 & -29.34 & 4.30 & $4 / 16$ \\
\hline Track - part 05 & 1.256 & -23.68 & 6.59 & $3 / 16$ \\
\hline Track - part 06 & 0.934 & -24.97 & 6.02 & $5 / 16$ \\
\hline Track - part 07 & 2.034 & -47.44 & 2.76 & $7 / 16$ \\
\hline Track - part 08 & 1.187 & -24.38 & 9.25 & $1 / 16$ \\
\hline$\sum a b s(\operatorname{Re}+j \mathrm{Im})_{01-08}$ & 9.321 & -24.77 & 5.64 & $1 / 16$ \\
\hline Track & 10 & -25.99 & 11.27 & $0 / 16$ \\
\hline
\end{tabular}

SNR ${ }^{*}$ - denotes: power of the spectral line " 1 " to power of the spectral line " 0 " ratio. Assumption: logical bit " 1 " is pair spectral lines mapped as a sequence " 1 " and " 0 ", logical bit " 0 " is pair spectral line mapped as a sequence " 0 " and " 1 ".

\section{Conclusions}

The proposed system proved the usefulness of the computer simulated HF/VHF radio link as well as of the preliminary radio link tests with FM/AM modulation. Watermark perceptual transparency at the host speech signal presence was confirmed in the experiment number 1 using subjective fidelity test ITU-R BS 1116-1. The watermark binary signature (PIN) is decoded correctly depending on the signal track's duration time and SMR value. Tracks with duration time of 10 seconds were decoded with 1 error in experiment number 2 (computer simulation), and 0 error in experiment number 3 (radio link). The steganographic system mode is used when the SSDS signal is embedded into the host signal. The 
phase drift scanner is a key element of this watermarking system and can be useful for providing the signal's coherency. The system is dedicated for radio correspondent authorisation. At present the described system is implemented on TI DSK 6713 for real-time processing.

\section{References}

[1] Nedeljko Cvejic, Tapo Seppänen, Spread spectrum audio watermarking using frequency hopping and attack characterization. Signal Processing, 84 (2004), pp.207-213, 2004.

[2] Zbigniew Piotrowski, Effective method of watermark embedding and decoding in the audio broadcast band. Military University of Technology, Warsaw, Poland, PhD dissertation, pp. 51-59, 2005

[3] Piotr Gajewski, Jerzy Łopatka, Zbigniew Piotrowski, A New method of frequency offset correction using coherent averaging. Journal of Telecommunications and Information Technology, 1/2005, pp. 142-146, 2005

[4] Methods for the Subjective Assessment of Small Impairments in Audio Systems Including Multichannel Sound Systems; Recommendation ITU-R BS.1116.

[5] Richard G. Lyons, Understanding Digital Signal Processing, Prentice Hall PTR Publication, 2004 